\title{
Overexpression of Angiogenic Factors and Matrix Metalloproteinases in the Saliva of Oral Squamous Cell Carcinoma Patients: Potential Non-Invasive Diagnostic and Therapeutic Biomarkers
}

\section{Meijuan Cai}

Affiliated Stomatology Hospital of Guangzhou Medical University

Zhichao Zheng

Affiliated Stomatology Hospital of Guangzhou Medical University

Zhibao Bai

Guangzhou First People's Hospital

Kexiong Ouyang

Affiliated Stomatology Hospital of Guangzhou Medical University

Qiuyu Wu

Affiliated Stomatology Hospital of Guangzhou Medical University

Shaofen Xu

Affiliated Stomatology Hospital of Guangzhou Medical University

Lihuan Huang

Affiliated Stomatology Hospital of Guangzhou Medical University

Yingtong Jiang

Hexian Memorial Affiliated Hospital of Southern Medical University

\section{Lijing Wang}

Vascular Biology Research Institute, Guangdong Pharmaceutical University

Jie Gao

Affiliated Stomatology Hospital of Guangzhou Medical University

Janak L. Pathak

Affiliated Stomatology Hospital of Guangzhou Medical University

Lihong Wu ( $\nabla$ wcanhong@163.com )

Affiliated Stomatology Hospital of Guangzhou Medical University

\section{Research Article}

Keywords: Angiogenic factors, diagnostic markers, MMPs, oral squamous cell carcinoma, saliva

Posted Date: July 6th, 2021 
DOl: https://doi.org/10.21203/rs.3.rs-646337/v1

License: (c) (1) This work is licensed under a Creative Commons Attribution 4.0 International License. Read Full License 


\section{Abstract}

Backgound: Salivary biomarkers hold huge potential for the non-invasive diagnosis of oral squamous cell carcinoma. Angiogenic factors and matrix-metalloproteinases (MMPs) are highly expressed in OSCC tissue, but their expression in the saliva is unknown. This study aimed to analyze the levels of angiogenic factors and MMPS in tumor-tissue and saliva of OSCC patients.

Methods: OSCC-tissue, adjacent normal tissue (ANT), saliva from OSCC patients, and healthy controls were obtained. The expression patterns of angiogenic factors and MMPs were analyzed by immunohistochemistry, protein chip array, and RT-qPCR.

Results: Results showed higher expression of ANG, ANG-2, HGF, PIGF, VEGF, MMP-1, MMP-2, MMP-3, MMP-8, MMP-9, MMP-10, MMP-13, TIMP-1, and TIMP-2 in OSCC-tissues compared to the ANT. Among the overexpressed markers in OSCC-tissues, HGF, VEGF, PIGF, PDGF-BB, MMP-1, MMP-3, MMP-8, MMP-9, MMP-10, MMP-13, and TIMP-2 were significantly upregulated in the saliva of OSCC patients compared to healthy controls.

Conclusions: The levels of HGF, VEGF, PIGF, PDGF-BB, MMP-1, MMP-3, MMP-8, MMP-9, MMP-10, MMP-13, and TIMP-2 were upregulated both in OSCC tissue and saliva of OSCC patients. Bioinformatic analysis revealed the correlation of these factors with patient survival and cancer functional states in head and neck cancer, indicating these factors as possible saliva-based non-invasive diagnostic/prognostic markers and therapeutic targets of OSCC.

\section{Introduction}

Oral squamous cell carcinoma (OSCC) is the most common oral cancer, accounting for $90 \%$ of all oral cancer [1]. Although many advances have been made in cancer treatment, the mortality rate of OSCC has remained unchanged. The 5 -year survival rate is around $50 \%$ in intermediate patients and less than $20 \%$ in advanced patients [2, 3]. Surgery, radiotherapy, and chemotherapy are currently available therapy for OSCCs [4]. Surgical resection is considered to be a promising treatment strategy for early cancer [5]. At present, the diagnosis of OSCC mainly relies on clinical examination, imaging examination, and histological examination of suspicious sites, but the lesion location of OSCC often occurs in hidden places and are not diagnosed until they have advanced or metastasized [6]. Therefore, new non-invasive therapeutic and diagnostic markers of OSCC with high sensitivity and specificity are in high demand [7].

Induced angiogenesis in OSCC had been reported to promote cancer progression and metastasis [8-11]. Vascular endothelial growth factor (VEGF), angiogenin (ANG), and platelet-derived growth factor (PDGF) signaling are reported as possible therapeutic targets in OSCC [12]. Similarly, angiogenic factors hepatocyte growth factor (HGF) and placental growth factor (PIGF) had been reported to regulate OSCC invasion [13-15]. Furthermore, Matrix metalloproteinases (MMPs) are a family of zinc ion and calcium ion dependent proteins, which promote tumor progression and metastasis [16]. Therefore, it is essential to investigate the role of angiogenic factors and MMPs in OSCC progression. 
In similar to blood, saliva is a complex body fluid known to contain several cellular and molecular components [17]. Saliva contacts directly with the oral mucosa and cancerous lesions thus are practicable for OSCC screening [18]. Detecting the altered level of certain molecules in saliva could be linked with oral and systemic diseases [19]. Saliva-based diagnosis is practical, non-invasive, is more accurate than available alternatives [20]. These advantages of saliva-based diagnosis may contribute to the early screening of many oral/systemic diseases and improve clinical management. It has been demonstrated that saliva is a useful diagnostic tool for assessing distant malignancies, including breast cancer [21], lung cancer [22], Sjögren syndrome [23]. Similarly, immunoglobulins have long been described as salivary biomarkers for HIV infection [20]. Several different salivary protein biomarkers have been reported. Interleukin (IL)-1, IL-6, IL-8, VEGF, and s90K/Mac-2 binding protein (M2BP), profilin, myeloid-related protein-14 (MRP14), and catalase have been reported as saliva biomarkers of oral cancer $[19,21-24]$. Various angiogenic factors and MMPs are reported to upregulate in OSCC tissue and serum. However, the expression pattern of angiogenic factors in the saliva of OSCC patient is not fully understood.

In this study, we aimed to evaluate the expression pattern of various angiogenic factors and MMPs in OSCC tissue and saliva of OSCC patients to unravel the possible non-invasive diagnostic/prognostic markers and therapeutic targets of OSCC.

\section{Materials And Methods}

\section{Patients and specimen collection}

OSCC and adjacent normal tissues (ANT) of 3 patients from the Affiliated Stomatology Hospital of Guangzhou Medical University and 7 patients from Guangzhou First People's Hospital were obtained. All patients received neither radiotherapy nor chemotherapy before surgery. ANT sample was obtained from $2 \mathrm{~cm}$ distance of the tumor tissue. Tissue specimens were stained with H\&E to distinguish cancerous tissue from ANT. Saliva samples were collected from OSCC patients without chemotherapy or radiotherapy. Volunteers were adjusted to regulatory sleep and required not to eat or drink within 1-2 $\mathrm{h}$ after brushing in the morning. Saliva was collected from 8 OSCC patients and 8 healthy control and stored at $-80^{\circ} \mathrm{C}$. Patient and healthy control clinical characteristics and demographics are shown in Table S1.

\section{Immunohistochemistry (IHC)}

OSCC and normal tissues were fixed with $4 \%$ paraformaldehyde and embedded in paraffin, $5 \mu \mathrm{m}$ thick sections of the specimens were deparaffinized and rehydrated in sequential xylene and gradient ethanol. The sections were immersed in citrate buffer ( $\mathrm{pH} \mathrm{6.0)}$ and heated for $30 \mathrm{~min}$ for antigen retrieval. After incubating with $3 \% \mathrm{H}_{2} \mathrm{O}_{2}$, the sections were blocked with bovine serum albumin (BSA, Serivicebio, China), followed by incubated with the primary antibodies CD31 (Serivicebio, China, 1:300), CK18 (Abcam, USA, 1:200), MMP2 (Serivicebio, China, 1:1200), MMP3 (Serivicebio, China, 1:800), and MMP13 (Serivicebio, 
China, 1:200) overnight at $4^{\circ} \mathrm{C}$. Sections were then washed three times with PBS and incubated with goat anti-rabbit IgG (Serivicebio, China, 1:200) with HRP at room temperature. The bound antibody was then observed with diaminoaniline (DAB, Serivicebio, China), and the sections were counterstained with hematoxylin. CD31 staining was used to assess microvascular density (MVD) following the method described previously [25].

\section{Real-time quantitative PCR (RT-qPCR) analysis}

Total RNA was extracted from human OSCC tissue and ANT samples using TRIzol reagent (Invitrogen, USA) according to the manufacturer's instructions. The cDNA was generated in the T100 gradient PCR machine (Bio-rad, USA) by using PrimeScript RT reagent kit with gDNA Eraser (Takara, Japan). RT-qPCR was performed using TB Green Premix Ex Taq II Kit (Takara, Japan) on an AriaMx Real-time quantitative PCR machine (Agilent, USA). The PCR reaction conditions were $95^{\circ} \mathrm{C}$ for $30 \mathrm{~s}$, followed by 40 cycles at $95^{\circ} \mathrm{C}$ for $5 \mathrm{~s}$ and $60^{\circ} \mathrm{C}$ for $30 \mathrm{~s}$. Each reaction was performed in triplicate. The expression of the GAPDH gene was used as the internal control. The fold change relative to the control group was measured by $2^{-}$ $\Delta \Delta \mathrm{Ct}$ method. Primer sequences used in this study were list in Table S2.

\section{Protein chip array}

Tissue and saliva samples from patients were used to measure the expression of angiogenin, angiopoietin-2 (ANG-2), basic fibroblast growth factor (bFGF), heparin-binding epidermal growth factor (HB-EGF), HGF, LEP, platelet-derived growth factor (PDGF-BB), PIGF, VEGF by the Quantibody ${ }^{\circledR}$ Human Angiogenesis Array 1 (RayBiotech, USA) and MMP-1, MMP-2, MMP-3, MMP-8, MMP-9, MMP-10, MMP-13, tissue inhibitors of MMPs (TIMP)-1, TIMP-2 by the Quantibody ${ }^{\circledR}$ Human MMP Array 1 (RayBiotech, USA). The detection was performed according to the manufacturer's instructions. Briefly, supernatants from centrifuged tissue samples treated with cell lysate and centrifuged saliva samples were used. The glass

slide was completely air dry and blocked by a blocking solution for $1 \mathrm{~h}$. The tissue sample diluent $100 \mu \mathrm{L}$ $(500 \mu \mathrm{g} / \mathrm{mL})$ and $80 \mu \mathrm{L}$ of the diluted tissue lysate or saliva samples were separately added to the slides and incubated overnight at $4^{\circ} \mathrm{C}$. The samples diluted were decanted from the wells and washed with wash buffer at room temperature with gentle rocking. The detection antibody cocktail was added to each well and incubated at room temperature, followed by a washing step. Afterward, Cy3 equivalent dyeconjugated streptavidin was added and incubated in a dark room, followed by a washing step.

Fluorescence signals were obtained by an InnoScan 300 Microarray Scanner (Innopsys, France) at 532 $\mathrm{nm}$ wavelength and $10 \mu \mathrm{m}$ resolution. Data were analyzed using Mapix (Innopsys, France) analysis software.

\section{Survival analysis}

The expression data of head and neck squamous carcinoma for angiogenic-related and MMPS was downloaded from the Kaplan Meier plotter (http://www.oncolnc.org/). The survival curve for 248 HNSCC patients with higher expression of angiogenic factors/MMPs and other 248 patients with lower angiogenic factors/MMPs expression was plotted to evaluate the 5-Year survival percentage and duration 
as described previously [26]. Log-rank (Mantel-Cox) test was used to evaluate the survival rate of significant difference.

\section{Correlation analysis of angiogenic factors and MMPs expression with the functional states in head and neck squamous cell carcinoma (HNSCC)}

Average correlations data between genes of interest and functional states in HNSCC patients were downloaded from CancerSEA (http://biocc.hrbmu.edu.cn/CancerSEA. CancerSEA is a dedicated database that aims to comprehensively decode distinct functional states of cancer cells at the single-cell level. The expression pattern of ANGs and MMPs and their correlation with 14 crucial biological functions in HNSCC, including cancer cell stemness, invasion, metastasis, proliferation, EMT, angiogenesis, apoptosis, cell cycle, cell differentiation, DNA damage, DNA repair, hypoxia, inflammation, and quiescence were analyzed.

\section{Statistical analysis}

The results were represented as the mean \pm standard deviation (SD). The statistical significance was determined by Student's t-test at $p<0.05$. The micrograph of IHC was taken using PRECICE 500B ScanScope image analyzer (UNIC, China). Image $\mathrm{J}$ software was used for calculating the positive area of IHC. The graphs were plotted using GraphPad Prism 7.0.

\section{Results}

\section{Histology and immunohistochemistry of angiogenic factors in ANT and OSCC tissues}

H\&E stained ANT histological tissue section showed normal oral tissue histology. In contrast, H\&E stained OSCC tissue section showed a distinct pattern of well-differentiated OSCC (Fig. 1A). CD31 immunohistochemistry tissue section showed few CD31-stained spots in ANT sections, and distinct CD31-stained microvessels in OSCC tissue sections (Fig. 1A). CK18 is a soluble cytokeratin present in proliferating cells and is highly concentrated in the G2-M phase of the cell cycle [27]. Since CK18 is highly expressed in OSCC tissues, we further performed CK18 immunohistochemistry. Brown granular staining in the nucleus of tumor cells was considered positive for CK18. ANT sections were hardly stained with CK18, but OSCC tissue sections showed intense CK18 staining (Fig. 1A). Quantitative analysis of CD31 stained microvessel structure revealed 2.5-fold higher MVD in OSCC tissue compared to ANT (Fig. 1B). Similarly, OSCC tissue showed a 5.06-fold higher expression of CK18 compared to ANT (Fig. 1C). mRNA expression analysis by RT-qPCR also showed upregulation of CD31 and CK18 in OSCC tissue compared to ANT (Fig. 1D and E).

\section{mRNA expression of angiogenic factors in OSCC tissues and ANT}

We further analyzed the mRNA level expression of angiogenic factors in OSCC and ANT tissue by RTqPCR. The expression of ANG, ANG-2, HGF, PIGF, VEGF, PDGF-BB, and HB-EGF in the OSCC-group were 1.58-, 13.56-, 3.00-, 8.86-, 3.34-, 2.08-, and 4.63-fold higher, respectively compared to ANT-group (Fig. 1F- 
1N). LEP expression in OSCC-group was not significantly affected compared to ANT-group (Fig. 10). The expression of bFGF was reduced in OSCC-group compared to ANT-group (Fig. 1H, 1M).

\section{Protein level expression of angiogenic factors in OSCC tissues and ANT}

We analyzed the protein level expression of angiogenic factors in OSCC and ANT tissue lysates by protein chip array. Among the angiogenic factors tested, the fluorescence intensities of angiogenin, ANG, ANG-2, HGF, PIGF, and VEGF were prominent in OSCC-group compared to ANT-group (Fig. 2A). Quantitative analysis revealed 1.80-, 6.37-, 2.21-, 3.47-, and 3.30-fold higher expression of ANG, ANG-2, HGF, PIGF, and VEGF in the OSCC-group, respectively compared to ANT-group (Fig. 2B-2G). Expressions of PDGF-BB, bFGF, HB-EGF, and leptin were not significantly changed in OSCC tissue compared to ANT (Fig. 2H-2K).

\section{Protein level expression of angiogenic factors in saliva of OSCC patients and healthy controls}

We further analyzed the protein level expression of angiogenic factors in the saliva of OSCC patients and healthy controls. The main aim of this part experiment was to further determine the potential application of angiogenic factors as non-invasive diagnostic and prognostic markers in OSCC. Among the angiogenic markers tested, 2.00-, 8.10-, and 1.38-, higher expression of HGF, PIGF, and VEGF, were upregulated in the saliva of OSCC patients compared to healthy controls (Fig. 3D-3H). Interestingly, PDGFBB was not detected in healthy controls, while $62.5 \%$ of OSCC patients showed higher expression of PDGF-BB in saliva (Fig. 3H). Expressions of ANG, ANG-2, bFGF, HB-EGF, and leptin in saliva were not significantly different in OSCC compared to control (Fig. 3A, 3B, 3I-K). Expressions of PIGF, PDGF-BB, bFGF, HB-EGF, and leptin in saliva relatively low to visualize the fluorescence intensity by the naked eye. However, fluorescence signals of these proteins were obtained by an InnoScan 300 Microarray Scanner and quantified.

\section{Immunohistochemistry of MMPs in ANT and OSCC tissues}

In addition, the expression pattern of MMPs in ANT and OSCC tissues was evaluated. MMP-2, MMP-3, and MMP-13 antibody-stained immunohistochemistry tissue section showed few brown stained spots in ANT sections, while distinct brown stained cell cytoplasm in OSCC tissue sections (Fig. 4A). Quantitative analysis of the staining showed higher expression of MMP-2, MMP-3, and MMP-13 in OSCC tissue than ANT (Fig. 4B, 4C, 4D).

\section{mRNA expression of MMPs in OSCC tissues and ANT}

We further analyzed the mRNA level expression of MMPs in OSCC and ANT tissue by RT-qPCR. The expressions of MMP-1, MMP-2, MMP-3, MMP-8, MMP-9, MMP-10, MMP-13, TIMP-1, and TIMP-2 in OSCCgroup were 58.01-, 7.92-, 87.54-, 7.69-, 37.51-, 146.29-, 115.20-, 7.60-, and 3.73-fold higher, respectively compared to ANT-group (Fig. 4E-4M).

Protein level expression of MMPs in OSCC tissues and ANT 
To determine the protein levels of MMPs in tissues, we performed a semi-quantitative protein chip array. The results showed that the fluorescence intensities of MMP-1, MMP-2, MMP3, MMP-8, MMP-9, MMP-10, MMP-13, TIMP-1, and TIMP-2 in the OSCC group were significantly increased compared with the ANTgroup (Fig. 5A). The mean fluorescence intensity of MMP-1, MMP-2, MMP3, MMP-8, MMP-9, MMP-10, MMP-13, TIMP-1 and TIMP-2 in OSCC tissue were 13.80-, 1.21-, 2.44-, 3.96-, 7.50-, 15.83-, 59.75-, 3.29-, and 1.89-fold higher than ANT-group, respectively (Fig. 5B-J). The result of protein level expression of MMPs was in accordance with the results of RT-qPCR (Fig. 4).

\section{Protein level expression of MMPs in saliva of OSCC patients and healthy controls}

The protein level expression of MMPs in the saliva of OSCC patients and healthy controls was further analyzed. It demonstrated that the expression levels of MMP-1, MMP-3, MMP-8, MMP-9, MMP-10 and MMP-13 in saliva were relatively high to visualize the green fluorescence (Fig. 6A). Quantification analysis revealed that the MMP-1, MMP-3, MMP-8, MMP-9, MMP-10, MMP-13, and TIMP-2 were 111.71-, 256.32-, 2.66-, 5.48-, 16.31-, 174.20-, 1.34-fold upregulated in the saliva of OSCC patients, respectively, compared to healthy controls (Fig. 6B, D-H and J). Intriguingly, MMP-1, MMP-3 and MMP-13 were highly expressed in the saliva of the OSCC group, but their expressions were too low to be detected in the healthy individual.

\section{The correlation between angiogenic factors and MMPs expression with the functional states in HNSCC}

The Figure 7 depicts the correlation between the expression patterns of ANGs and MMPs expression with the functional states in HNSCC. ANG2 and PIGF expression positively correlate with cancer cell stemness. HB-EGF expression positively correlates with inflammation, cancer cell quiescence and stemness. The expression of leptin positively correlates with cancer cell stemness. MMPs expression was positively correlated with angiogenesis, EMT and metastasis.

\section{Survival analysis}

We further analyzed the survival curve from 124 248 HNSCC patients (25 50\%) with higher expression of ANGs and MMPs and 124 248 patients (25 50\%) with lower expression of ANGs and MMPs. HNSCC patients with lower expression of ANG ( $p=0.0094)$, PIGF $(p=0.0426)$, PDGF-BB $(p=0.0285)$, HB-EGF $(p=0.0091)$, MMP-8 $(p=0.0214)$ and TIMP-1 $(p=0.0023)$ showed significantly higher 5 years survival rate compared to those HNSCC patients with higher expression of these factors (Fig. 8A, D, F, H, M, Q). Similarly, HNSCC patients with lower expression of MMP-1 ( $p=0.1056)$, MMP-2 ( $p=0.3013)$, MMP-3 $(p=0.2857)$, MMP-9 $(p=0.1032)$, and TIMP-2 $(p=0.1719)$ showed higher 5 years survival rate compared to those HNSCC patients with higher expression of these factors but there was no significant different between lower and higher groups (Fig. 8J, K, L, R).

\section{Discussion}


Identification of novel biomarkers for OSCC diagnosis/prognosis helps to early diagnosis and treatment and decreases patient morbidity and mortality. Considering the importance of angiogenesis and MMPs in OSCC development and metastasis, it is wise to explore the expression pattern of angiogenic factors and MMPs. Results of this study found a direct correlation of angiogenesis with OSCC development. This study found a higher expression of HGF, VEGF, PIGF, PDGF-BB, MMP-1, MMP-3, MMP-8, MMP-9, MMP-10, MMP-13, and TIMP-2 both in OSCC tissue and saliva of OSCC patients. Our findings indicate these factors as saliva-based non-invasive possible diagnostic/prognostic markers and therapeutic targets of OSCC (Fig. 9).

Histological analysis is the gold standard for OSCC diagnosis. No tumor progression in ANT-tissues was verified by histological analysis. CD31, an endothelial marker, is highly expressed in various cancer tissues, including OSCC $[28,29]$. In this study, CD31 expression was robustly upregulated in OSCC tissues compared to ANT. The higher degree of angiogenesis promotes not only OSCC progression but also cancer invasion [30]. CK18, a cytokeratin protein, is highly expressed in proliferating cancer cells, including OSCC [31]. We found higher MVD in OSCC tissue compared to ANT. Similarly, the expression pattern of CK18 was in accordance with the higher CD31 expression and angiogenesis in OSCC. These findings indicate the key role of angiogenesis in OSCC progression.

ANG, ANG-2, HGF, PIGF, PDGF-BB, HB-EGF, VEGF, bFGF, and leptin are key growth factors that regulate angiogenesis in pathophysiological conditions [32]. In this study, we evaluated the expression of all these angiogenic growth factors in OSCC tissue and saliva of OSCC patients. The mRNA levels of ANG, ANG-2, HGF, PIGF, PDGF-BB, HB-EGF, and VEGF were upregulated in OSCC tissues compared to paired-ANT. Furthermore, the protein levels of ANG, ANG-2, HGF, PIGF, and VEGF were significantly increased in OSCC tissues. Saliva possesses tremendous potential in disease diagnosis due to its critical advantages, including minimum cost, non-invasiveness, and easy collection [33]. HGF, PIGF, and VEGF had been reported as therapeutic targets in OSCC [13-15, 34]. HGF regulates gastric cancer progression and metastasis [35]. In various cancers, PIGF is involved in tumor immune escape and metastasis [36]. PolzDacewicz and colleagues had reported the higher expression of VEGF in serum and saliva of oropharyngeal squamous cell carcinoma patients [37]. PDGF-BB is overexpressed in OSCC tissues and correlates with the tumorigenesis and poor prognosis of OSCC [38]. In this study, HGF, PIGF, VEGF, and PDGF-BB were found to be significantly upregulated in OSCC tissue as well as in the saliva of the patients. Future research on the role of HGF, PIGF, VEGF, and PDGF-BB in OSCC progression, and metastasis could be useful to unravel these factors as novel diagnostic and therapeutic markers. This is the first study to show the higher expression of HGF, PIGF, and PDGF-BB in the saliva of OSCC patients. Our findings indicate that the angiogenic markers HGF, PIGF, VEGF, and PDGF-BB could be saliva-based novel diagnostic markers of OSCC.

MMPs participate in tumor progression, and metastasis, thereby hold diagnostic and therapeutic applications potential $[39,40]$. MMP-1/protease-activated receptor-1 (PAR1) signaling axis plays an essential role in tumor angiogenesis by inducing vascular permeability [41]. Serum and salivary MMP1, MMP2, MMP-9, MMP-10, MMP-12, and MMP13 had been reported to upregulate in OSCC [42-45]. 
Moreover, MMP-9 triggers the angiogenic switch by releasing VEGF during tumorigenesis [46]. We found that the mRNA and protein levels of MMP-1, MMP-2, MMP-3, MMP-8, MMP-9, MMP-10, MMP-13, TIMP-1, and TIMP-2 were significantly increased in OSCC tissues compared to paired-ANT. Furthermore, MMP-1, MMP-3, MMP-8, MMP-9, MMP-10, MMP-13, and TIMP-2 were significantly upregulated in the saliva of OSCC patients compared to the healthy ones. Accordingly, the expression of MMP-1, MMP-3, MMP-8, MMP-9, MMP-10, MMP-13, and TIMP-2 were upregulated both in OSCC tissue and in the saliva of OSCC patient. MMP-1, MMP-2, MMP-3, MMP-9, MMP-13, TIMP-1, and TIMP-2 had been reported to overexpress in head and neck cancer tissues compared with normal tissues. Thus, our data is consistent with previous reports $[47,48]$. This is the first study to report the overexpression of salivary MMP-3, MMP-8, and TIMP-2 in OSCC suggesting these markers as saliva-based potential novel diagnostic/prognostic markers as well as therapeutic targets of OSCC.

In this study, we re-confirmed higher angiogenesis in human OSCC tissues. We analyzed the expression pattern of a panel of angiogenic factors and MMPs in OSCC tissue and saliva of OSCC patients. RT-qPCR, immunohistochemistry, and protein chip array analysis were used to extensively analyze the expression of aforementioned markers in mRNA and protein levels in OSCC tissue and saliva. This study revealed the overexpression of HGF, PIGF and PDGF-BB, MMP-3, MMP-8, and TIMP-2 both in serum and saliva of OSCC patients for the first time. Moreover, the survival analysis of HNSCC showed a direct correlation with the higher expression of the ANG, PIGF, PDGF-BB, HB-EGF, MMP-8, and TIMP-1 expression with the lower survival rate of the patients. Moreover, the expression patterns of MMPs and TIMP-1 in HNSCC positively correlated with angiogenesis, EMT, and metastasis. Although these are the results from HNSCC patients, the similarity between HNSCC and OSCC provides a hint about the functions of ANGs and MMPs in OSCC. The survival analysis and cancer cell functional states correlation with ANGs/MMPs expression are needed to confirm the exact roles of ANGs and MMPs in OSCC development and progression.

The overexpression of VEGF, MMP-1, MMP-2, MMP-3, MMP-9, MMP-10, and MMP-13 in OSCC tissue and saliva corroborates the findings of the previous studies [42-48]. Sample inclusion from a small number OSCC patients is a limitation of this study. A multicenter study with higher patient numbers is recommended to further validate the findings of this study. Since the higher levels of salivary HGF, PIGF, PDGF-BB, MMP-3, MMP-8, and TIMP-2 are first time reported, their role as diagnostic and prognostic markers, and therapeutic targets for OSCC should be thoroughly investigated.

\section{Conclusions}

In conclusion, this study showed higher expression of angiogenesis factors and MMPs in OSCC tissues and saliva of OSCC patients. Among the angiogenic factors tested, HGF, PIGF, VEGF, and PDGF-BB were significantly overexpressed in OSCC tissues and saliva. Similarly, among the MMPs tested, the MMP-1, MMP-3, MMP-8, MMP-9, MMP-10, MMP-13, and TIMP-2 were significantly overexpressed in OSCC tissues and saliva. mRNA and protein level overexpression of angiogenic factors and MMPs in OSCC tissues corroborates the protein level overexpression of these factors in saliva. Among all the overexpressed 
factors in OSCC tissues and saliva, this study first time reveals the overexpression of salivary HGF, PIGF, PDGF-BB, MMP-3, MMP-8, and TIMP-2 in OSCC suggesting these factors as possible saliva-based noninvasive diagnostic and therapeutic biomarkers of OSCC.

\section{List Of Abbreviations}

OSCC

oral squamous cell carcinoma

VEGF

vascular endothelial growth factor

ANG

angiogenin

PDGF

platelet-derived growth factor

HGF

hepatocyte growth factor

PIGF

placental growth factor

MMPs

matrix metalloproteinases

M2BP

Mac-2 binding protein

MRP14

myeloid-related protein-14

ANT

adjacent normal tissues

MVD

microvascular density

HNSCC

head and neck squamous cell carcinoma

\section{Declarations}

\section{Ethics and approval and consent to participate}

The study was approved by the medical ethics comittee of Affiliated Stomatology Hospital of Guangzhou Medical University (Approval no. KY2019026).

\section{Consent for publication}

The consents for publication were acquired from all the participants. 
Availability of data and materials

The datasets used and analyzed during the current study are available from the corresponding author on reasonable request.

\section{Competing interests}

The authors declare that they have no competing interest.

\section{Fundings:}

This research was funded by National Natural Science Foundation of China (31801152), University Student Laboratory Open Project of Guangzhou Medical University (2020-27), and Innovation and Entrepreneurship Training program for College Students of Guangzhou Medical University (2019A049).

\section{Authors' contrubitions}

LW, PJL and GJ designed the project, interpretated the data, drafted the work and substantively revized the work. MC, ZZ, ZB, KOY, QW, SX, LH, YJ, and LJW acquired and analyzed the data. All authors read and approved the final manuscript.

\section{References}

1. Chen TW, Lee C-C, Liu H, Wu C-S, Pickering CR, Huang P-J, Wang J, Chang IY-F, Yeh Y-M, Chen C-D: APOBEC3A is an oral cancer prognostic biomarker in Taiwanese carriers of an APOBEC deletion polymorphism. Nature Communications, 2017, 8(1):465.

2. Bloebaum $M$, Poort $L$, Böckmann R, Kessler P: Survival after curative surgical treatment for primary oral squamous cell carcinoma. Journal of Cranio-Maxillo-Facical Surgery, 2014, 42(8):1572-1576.

3. Hasegawa H, Kusumi Y, Asakawa T, Maeda M, Oinuma T, Furusaka T, Oshima T, Esumi M: Expression of von Hippel-Lindau tumor suppressor protein ( $\mathrm{pVHL}$ ) characteristic of tongue cancer and proliferative lesions in tongue epithelium. BMC Cancer, 2017, 17(1):381.

4. Reis PP, Waldron L, Perez-Ordonez B, Pintilie M, Galloni NN, Xuan Y, Cervigne N, Warner GC, Makitie AA, Simpson C: A gene signature in histologically normal surgical margins is predictive of oral carcinoma recurrence. BMC Cancer, 2011, 11(1):437.

5. Kummerow KL, Du L, Penson DF, Shyr Y, Hooks MA: Nationwide trends in mastectomy for early-stage breast cancer. JAMA Surgery, 2015, 150(1):9-16.

6. Mehrotra R, Yadav S: Oral squamous cell carcinoma: etiology, pathogenesis and prognostic value of genomic alterations. Indian Journal of Cancer, 2006, 43(2):60-66.

7. Jia-Yo W, Chen Y, Ho-Ren C, Duen-Jeng W, Wen-Chien C, Sheng-Yang L, Che-Tung L, Yueh-Chao Y, WeiChung Vivian Y: Potential biomarkers in saliva for oral squamous cell carcinoma. Oral Oncology, 2010, 46(4):226-231. 
8. Kalra M, Rao N, Nanda K, Rehman F, Girish KL, Tippu S, Arora A: The role of mast cells on angiogenesis in oral squamous cell carcinoma. Medicina Oral Patologia Oral Y Cirugia Bucal 2012, 17(2):E190-E196.

9. Kabiraj A, Jaiswal R, Singh A, Gupta J, Singh A, Samadi FM: Immunohistochemical evaluation of tumor angiogenesis and the role of mast cells in oral squamous cell carcinoma. Journal of Cancer Research and Therapeutics, 2018, 14(3):495-502.

10. Yoshida S, Kawai H, Eguchi T, Sukegawa S, Oo MW, Anqi C, Takabatake K, Nakano K, Okamoto K, Nagatsuka H: Tumor Angiogenic Inhibition Triggered Necrosis (TAITN) in Oral Cancer. Cells, 2019, 8(7):E761.

11. Cui Z, Liu QL, Sun SQ, Jiao K, Liu DR, Zhou XC, Huang L: MiR-378a-5p inhibits angiogenesis of oral squamous cell carcinoma by targeting KLK4. Neoplasma, 2020, 67(1):85-92.

12. Chen L, Hu GF: Angiogenin-mediated ribosomal RNA transcription as a molecular target for treatment of head and neck squamous cell carcinoma. Oral Oncology, 2010, 46(9):648-653.

13. Hanzawa M, Shindoh M, Higashino F, Yasuda M, Inoue N, Hida K, Ono M, Kohgo T, Nakamura M, Notani $\mathrm{K}$ et al: Hepatocyte growth factor upregulates E1AF that induces oral squamous cell carcinoma cell invasion by activating matrix metalloproteinase genes. Carcinogenesis, 2000, 21(6):1079-1085.

14. Bu J, Bu X, Liu B, Chen F, Chen P: Inhibition of metastasis of oral squamous cell carcinoma by antiPLGF treatment. Tumour Biology, 2015, 36(4):2695-2701.

15. Xuan K, Li B, Guo H, Sun W, Kou XX, He XN, Zhang YJ, Sun J, Liu AQ, Liao L et al: Deciduous autologous tooth stem cells regenerate dental pulp after implantation into injured teeth. Sci Transl Med 2018, 10(455).

16. Coussens LM, Fingleton B, Matrisian LM: Matrix metalloproteinase inhibitors and cancer: trials and tribulations. Science 2002, 295(5564):2387-2392.

17. Rehak NN, Cecco SA, Csako G: Biochemical composition and electrolyte balance of "unstimulated" whole human saliva. Clinical Chemistry Laboratory Medicine, 2000, 38(4):335-343.

18. Yakob M, Fuentes L, Wang MB, Abemayor E, Wong DTW: Salivary biomarkers for detection of oral squamous cell carcinoma - current state and recent advances Current Oral Health Reports, 2014, $1(2): 133-141$.

19. Korostoff A, Reder L, Masood R, Sinha UK: The role of salivary cytokine biomarkers in tongue cancer invasion and mortality. Oral Oncology, 2011, 47(4):282-287.

20. Bonne NJ, Wong DTW: Salivary biomarker development using genomic, proteomic and metabolomic approaches. Genome Medicine, 2012, 4(10):82.

21. Bigler LR, Streckfus CF, Copeland L, Burns R, Dai X, Kuhn M, Martin P, Bigler SA: The potential use of saliva to detect recurrence of disease in women with breast carcinoma. Journal of Oral Pathology \& Medicine, 2002, 31(7):421-431.

22. Zhang L, Xiao H, Zhou H, Santiago S, Lee JM, Garon EB, Yang J, Brinkmann O, Yan X, Akin D: Development of transcriptomic biomarker signature in human saliva to detect lung cancer. Cellular 
and Molecular Life Sciences, 2012, 69(19):3341-3350.

23. Hu S, Gao K, Pollard R, Arellano-Garcia M, Zhou H, Zhang L, Elashoff D, Kallenberg CG, Vissink A, Wong DT: Preclinical validation of salivary biomarkers for primary Sjögren's syndrome. Arthritis Care \& Research, 2010, 62(11):1633-1638.

24. Hu S, Arellano M, Boontheung P, Wang J, Zhou H, Jiang J, Elashoff D, Wei R, Loo JA, Wong DT: Salivary proteomics for oral cancer biomarker discovery. Clinical Cancer Research, 2008, 14(19):6246-6252.

25. Weidner N, Semple JP, Welch WR, Folkman J: Tumor angiogenesis and metastasis-correlation in invasive breast carcinoma. The New England Journal of Medicine, 1991, 324(1):1-8.

26. Wang L, Chen Y, Yan Y, Guo X, Fang Y, Su Y, Wang L, Pathak JL, Ge L: miR-146a Overexpression in Oral Squamous Cell Carcinoma Potentiates Cancer Cell Migration and Invasion Possibly via Targeting HTT. Front Oncol 2020, 10:585976.

27. Chou CF, Riopel CL, Rott LS, Omary MB: A significant soluble keratin fraction in 'simple' epithelial cells. Lack of an apparent phosphorylation and glycosylation role in keratin solubility. Journal of Cell Science, 1993, 105(2):433-444.

28. Muller AM, Hermanns MI, Skrzynski C, Nesslinger M, Muller KM, Kirkpatrick CJ: Expression of the endothelial markers PECAM-1, vWf, and CD34 in vivo and in vitro. Experimental and Molecular Pathology, 2002, 72(3):221-229.

29. lamaroon A, Pongsiriwet S, Jittidecharaks S, Pattanaporn K, Prapayasatok S, Wanachantararak S: Increase of mast cells and tumor angiogenesis in oral squamous cell carcinoma. Journal of Oral Pathology \& Medicine, 2003, 32(4):195-199.

30. Sappayatosok K, Maneerat Y, Swasdison S, Viriyavejakul P, Dhanuthai K, Zwang J, Chaisri U: Expression of pro-inflammatory protein, iNOS, VEGF and COX-2 in oral squamous cell carcinoma (OSCC), relationship with angiogenesis and their clinico-pathological correlation. Medicina Oral Patologia Oral Y Cirugia Bucal, 2009, 14(7):E319-E324.

31. Nanda KD, Ranganathan K, Devi U, Joshua E: Increased expression of CK8 and CK18 in leukoplakia, oral submucous fibrosis, and oral squamous cell carcinoma: an immunohistochemistry study. Oral Surgery Oral Medicine Oral Pathology Oral Radiology, 2012, 113(2):245-253.

32. Esposito E, Aldrees S, Mastromonaco C, Zoroquiain P, Vila N, Logan PT, Hari S, Burnier MN: Evaluation of nicotinamide as an anti-inflammatory and anti-angiogenic agent in uveal melanoma cell lines. Arquivos Brasileiros De Oftalmologia, 2017, 80(2):74-77.

33. Rapado-Gonzalez O, Martinez-Reglero C, Salgado-Barreira A, Takkouche B, Lopez-Lopez R, SuarezCunqueiro MM, Muinelo-Romay L: Salivary biomarkers for cancer diagnosis: a meta-analysis. Annals of Medicine, 2020, 14:1-20.

34. Ferrara N: VEGF as a therapeutic target in cancer. Oncology, 2005, 69 (3):11-16.

35. Ding XS, Ji J, Jiang JL, Cai Q, Wang C, Shi M, Yu YY, Zhu ZG, Zhang J: HGF-mediated crosstalk between cancer-associated fibroblasts and MET-unamplified gastric cancer cells activates coordinated tumorigenesis and metastasis. Cell Death Dis 2018, 9. 
36. Albonici L, Giganti MG, Modesti A, Manzari V, Bei R: Multifaceted Role of the Placental Growth Factor (PIGF) in the Antitumor Immune Response and Cancer Progression. Int J Mol Sci 2019, 20(12).

37. Polz-Dacewicz M, Strycharz-Dudziak M, Dworzanski J, Stec A, Kocot J: Salivary and serum IL-10, TNF-alpha, TGF-beta, VEGF levels in oropharyngeal squamous cell carcinoma and correlation with HPV and EBV infections. Infect Agent Cancer 2016, 11:45.

38. Lin LH, Lin JS, Yang CC, Cheng HW, Chang KW, Liu CJ: Overexpression of Platelet-Derived Growth Factor and Its Receptor Are Correlated with Oral Tumorigenesis and Poor Prognosis in Oral Squamous Cell Carcinoma. Int J Mol Sci 2020, 21(7).

39. Malemud CJ: Matrix metalloproteinases (MMPs) in health and disease: an overview. Front Biosci 2006, 11:1696-1701.

40. Gonzalez-Avila G, Sommer B, Mendoza-Posada DA, Ramos C, Garcia-Hernandez AA, Falfan-Valencia $\mathrm{R}$ : Matrix metalloproteinases participation in the metastatic process and their diagnostic and therapeutic applications in cancer. Crit Rev Oncol Hematol 2019, 137:57-83.

41. Juncker-Jensen A, Deryugina El, Rimann I, Zajac E, Kupriyanova TA, Engelholm LH, Quigley JP: Tumor MMP-1 activates endothelial PAR1 to facilitate vascular intravasation and metastatic dissemination. Cancer Res 2013, 73(14):4196-4211.

42. Feng Y, Li Q, Chen J, Yi P, Xu X, Fan Y, Cui B, Yu Y, Li X, Du Y et al: Salivary protease spectrum biomarkers of oral cancer. Int J Oral Sci 2019, 11(1):7.

43. Peisker A, Raschke GF, Fahmy MD, Guentsch A, Roshanghias K, Hennings J, Schultze-Mosgau S: Salivary MMP-9 in the detection of oral squamous cell carcinoma. Med Oral Patol Oral Cir Bucal 2017, 22(3):e270-275.

44. Hema Shree K, Ramani P, Sherlin H, Sukumaran G, Jeyaraj G, Don KR, Santhanam A, Ramasubramanian A, Sundar R: Saliva as a Diagnostic Tool in Oral Squamous Cell Carcinoma - a Systematic Review with Meta Analysis. Pathol Oncol Res 2019, 25(2):447-453.

45. Nosratzehi T, Alijani E, Moodi M: Salivary MMP-1, MMP-2, MMP-3 and MMP-13 Levels in Patients with Oral Lichen Planus and Squamous Cell Carcinoma. Asian Pac J Cancer Prev 2017, 18(7):19471951.

46. Bergers G, Brekken R, McMahon G, Vu TH, Itoh T, Tamaki K, Tanzawa K, Thorpe P, Itohara S, Werb Z et al: Matrix metalloproteinase-9 triggers the angiogenic switch during carcinogenesis. Nat Cell Biol 2000, 2(10):737-744.

47. Vincent-Chong VK, Salahshourifar I, Karen-Ng LP, Siow MY, Kallarakkal TG, Ramanathan A, Yang YH, Khor GH, Rahman ZA, Ismail SM et al: Overexpression of MMP13 is associated with clinical outcomes and poor prognosis in oral squamous cell carcinoma. ScientificWorldJournal 2014, 2014:897523.

48. P OC, Rhys-Evans PH, Eccles SA: Expression of matrix metalloproteinases and their inhibitors correlates with invasion and metastasis in squamous cell carcinoma of the head and neck. Arch Otolaryngol Head Neck Surg 2001, 127(7):813-820. 

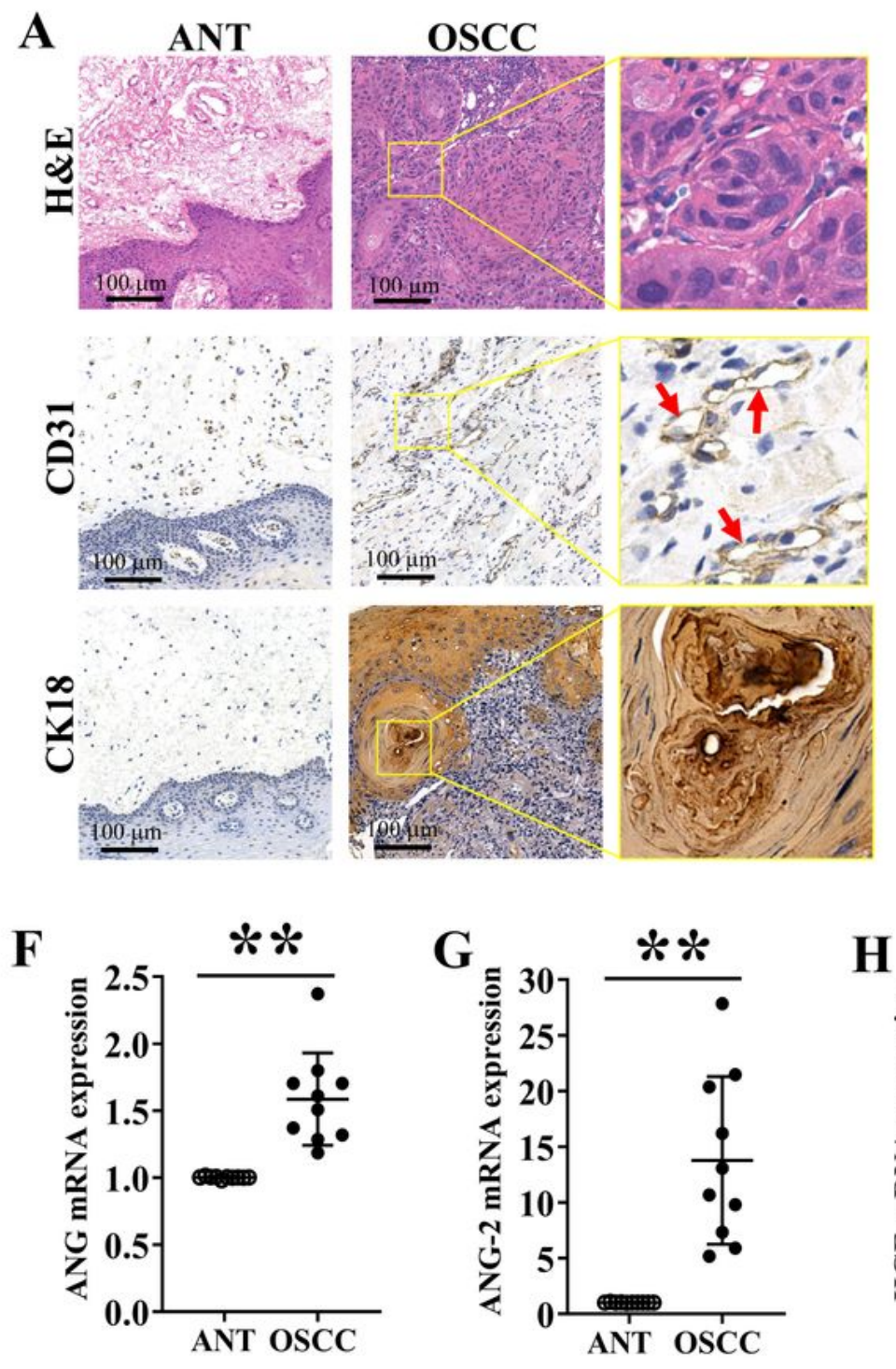


Figure 1

(A) H\&E-stained histological as well as CD31 and CK18 immunohistochemistry (IHC) images of human oral squamous cell carcinoma (OSCC) tissue sections and adjacent normal tissue (ANT). (B) Quantitative analysis of the microvascular density (MVD) in the CD31-stained area. (C) CK18 positive area in ANT and 
OSCC tissue (n=3). Relative mRNA expression of (D) CD31, (E) CK18, (F) angiogenin, (G) ANG-2, (H) HGF, (I) PIGF, (J), VEGF, (K) PDGF-BB, (L) bFGF, (M) HB-EGF, and (N) leptin in ANT and OcSS tissue $(n=10)$. Data are presented as mean \pm SD. Significant differences compared to the ANT group, ${ }^{*} p<0.05$ and $\star \star p<0.01$.

A

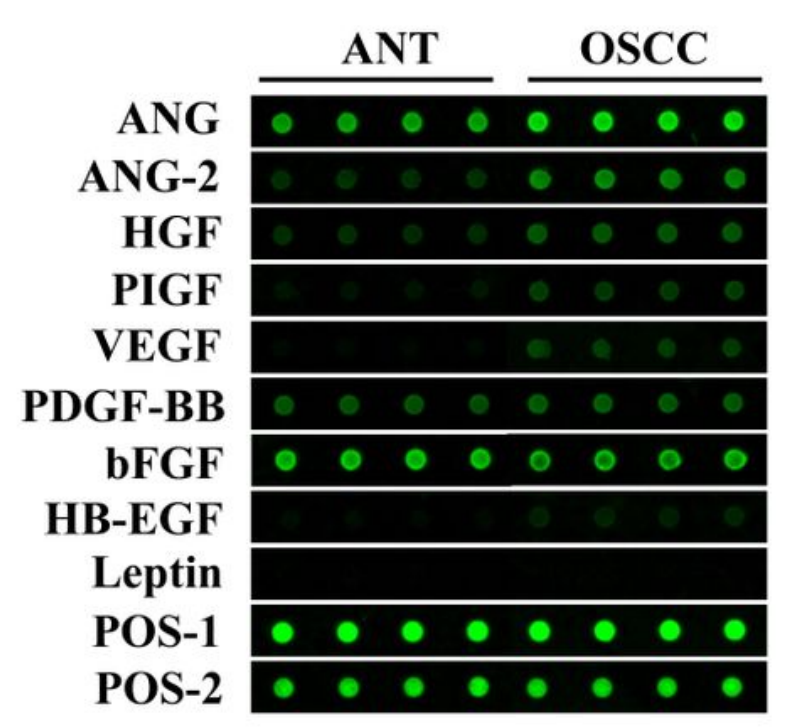

B
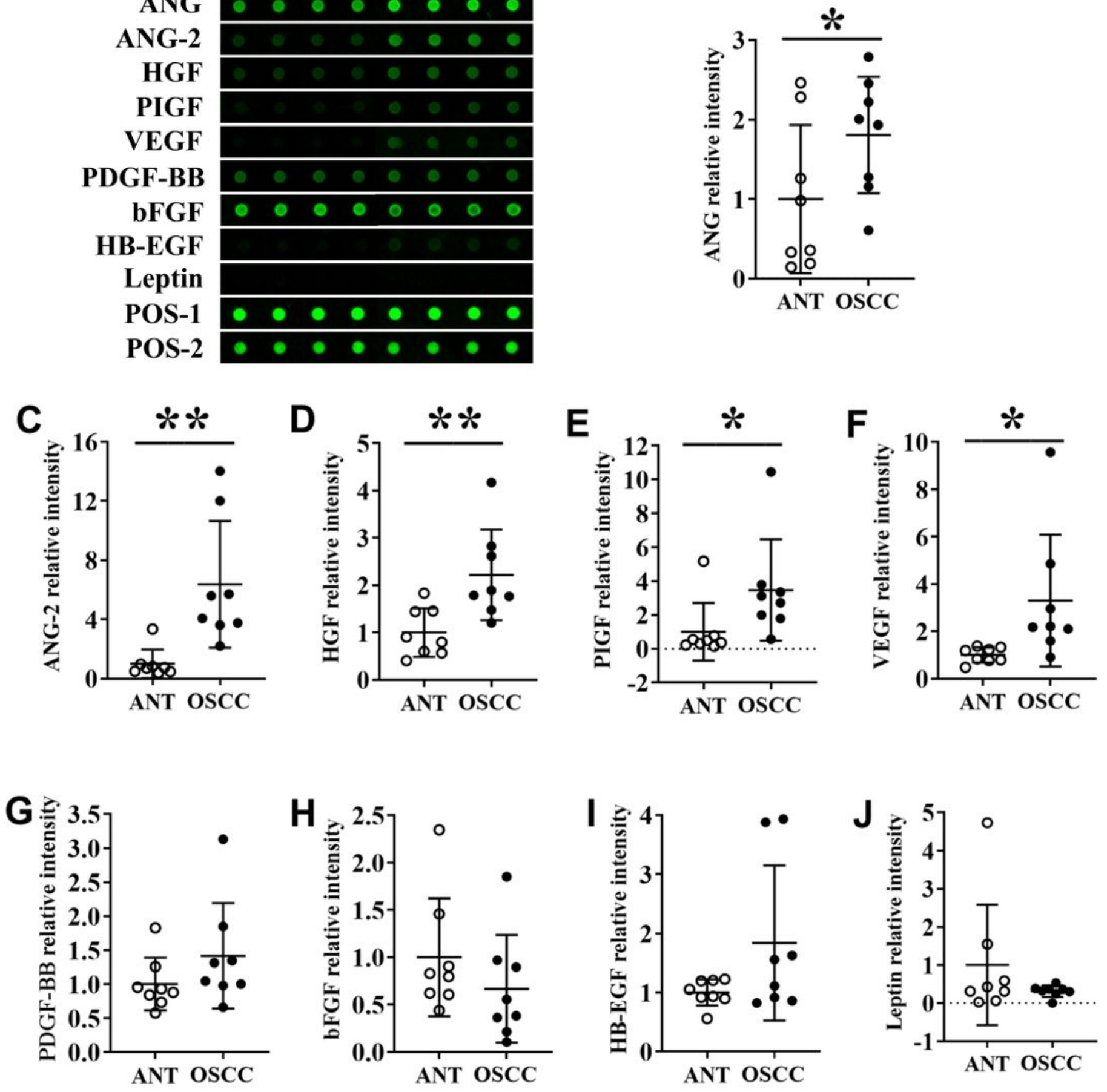

Figure 2 
(A) Representative protein array images showing the expression pattern of angiogenesis factors in adjacent normal tissue (ANT) and oral squamous cell carcinoma (OCSS) tissue lysates. Quantitative analysis of (B) angiogenin, (C) ANG-2, (D) HGF, (E) PIGF, (F) VEGF, (G) PDGF-BB, (H) bFGF, (I) HB-EGF, and $(J)$ leptin expression from protein array images. Data are presented as mean $\pm S D, n=8$. Significant differences compared with ANT group, ${ }^{*} p<0.05$ and ${ }^{* *} p<0.01$.
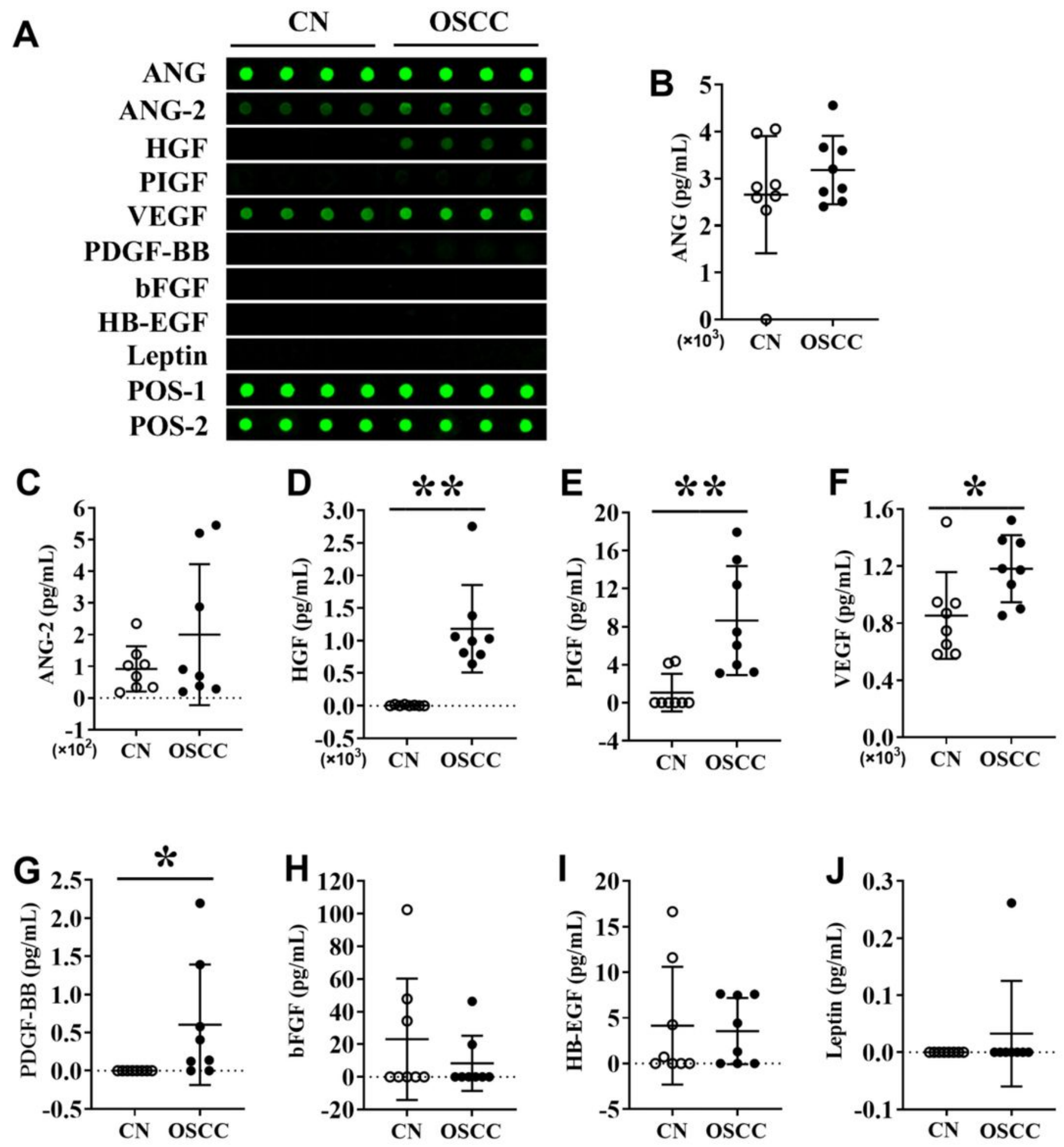

Figure 3 
(A) Representative protein array images showing the expression pattern of angiogenesis factors in the saliva of healthy controls and OSCC patients. Quantitative analysis of (B) angiogenin, (C) ANG-2, (D) HGF, (E) PIGF, (F) VEGF, (G) PDGF-BB, (H) bFGF, (I) HB-EGF, and (J) leptin expression from protein array images. Data are presented as mean $\pm S D, n=8$. Significant differences compared with the healthy control group, ${ }^{*} p<0.05$ and ${ }^{* *} p<0.01$.

A

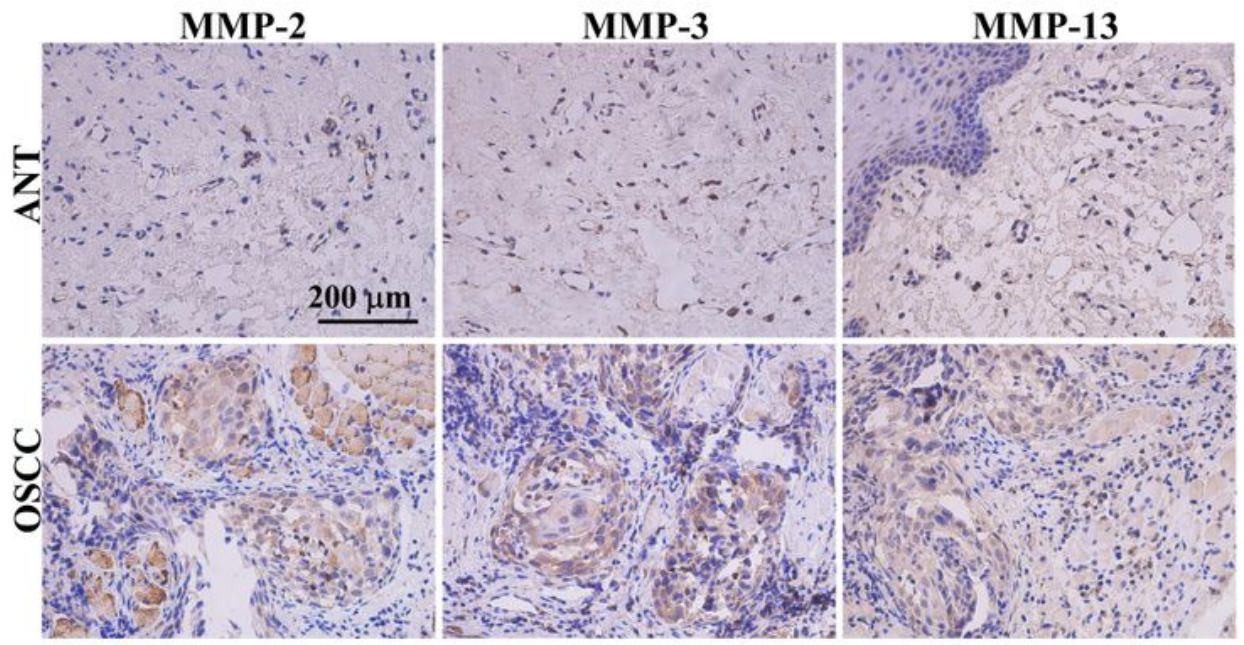

B

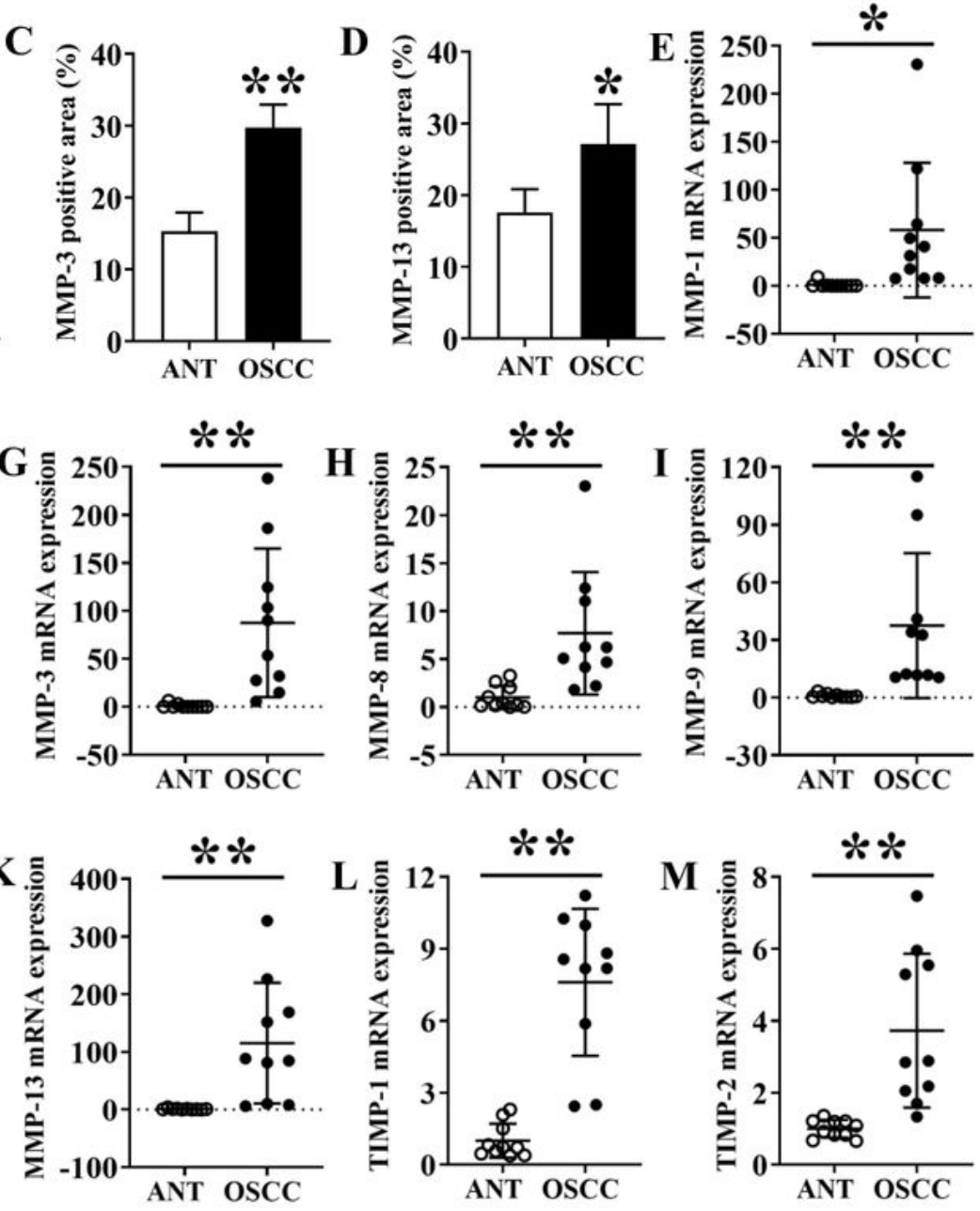

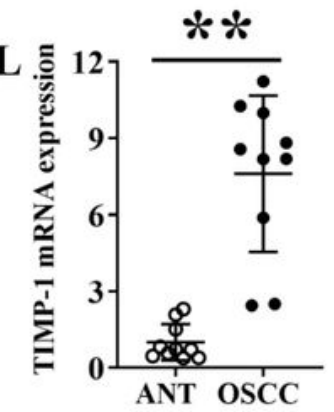

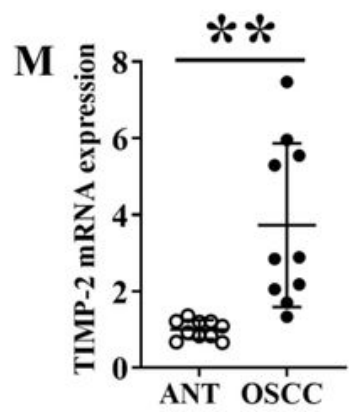

Figure 4 
(A) MMP-2, MMP-3, and MMP-13 immunohistochemistry (IHC) images of ANT and OSCC tissue sections. Quantification of (B) MMP-2, (C) MMP-3, and (D) MMP-13 positive area in ANT and OSCC tissue sections, $\mathrm{n}=3$. Relative mRNA expression of (E) MMP-1, (F) MMP-2, (G) MMP-3, (H) MMP-8, (I) MMP-9, (J) MMP-10, (K) MMP-13, (L) TIMP-1, and (M) TIMP-2 in ANT and OCSS tissue. Data are presented as mean \pm SD, $\mathrm{n}=10$. Significant differences compared with the ANT group, ${ }^{*} \mathrm{p}<0.05$ and ${ }^{*} \mathrm{p}<0.01$.

A
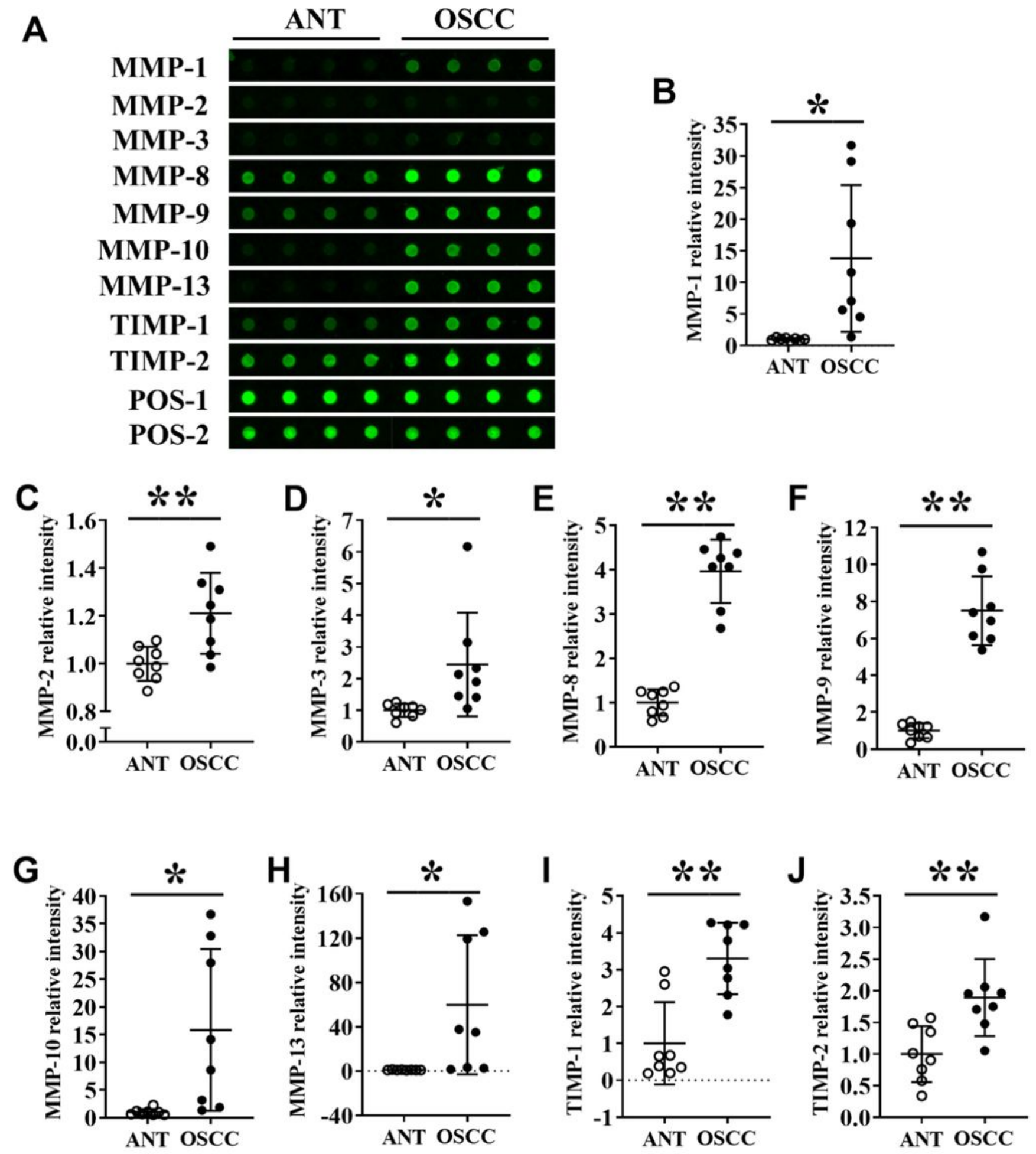

Figure 5 
(A) Representative protein array images showing the expression pattern of MMPs in ANT and OCSS tissue lysates. Quantitative analysis of (B) MMP-1, (C) MMP-2, (D) MMP-3, (E) MMP-8, (F) MMP-9, (G) MMP-10, (H) MMP-13, (I) TIMP-1, and (J) TIMP-2 expression from protein array images. Data are presented as mean $\pm S D, n=8$. Significant differences compared with ANT group, ${ }^{*} p<0.05$ and ${ }^{* *} p<0.01$

A

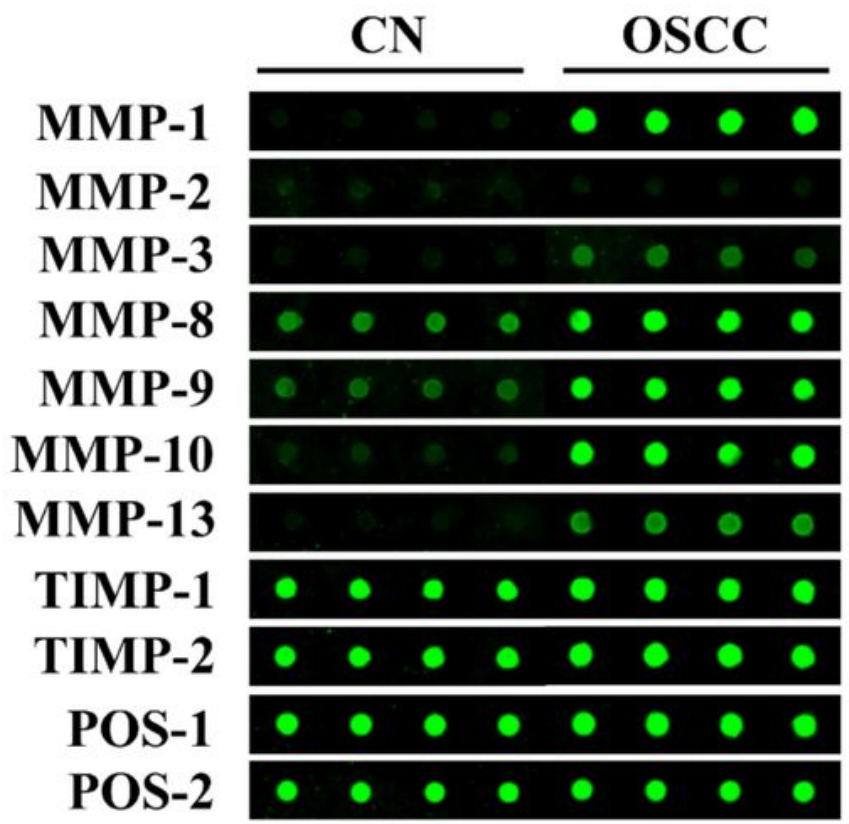

B
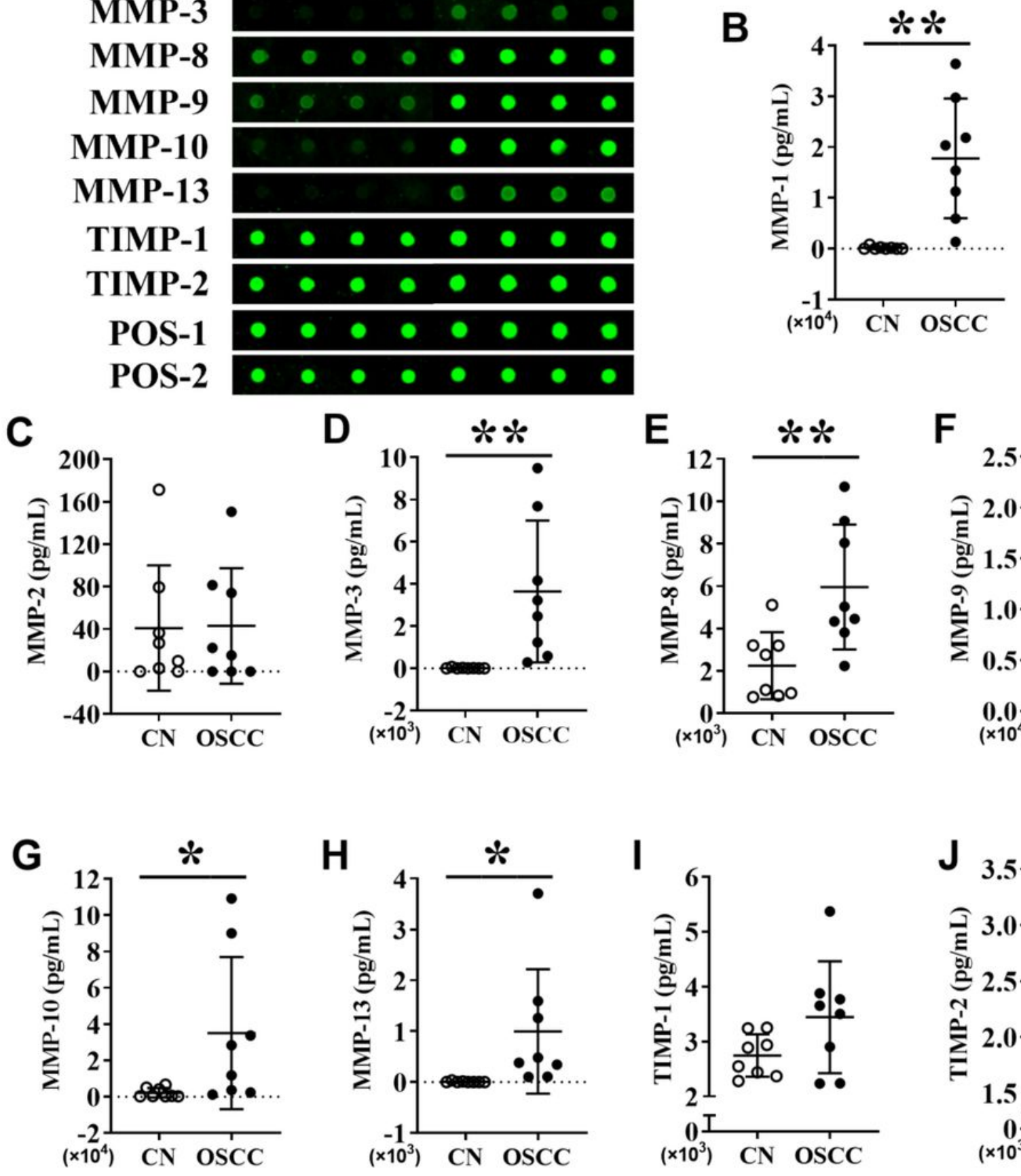
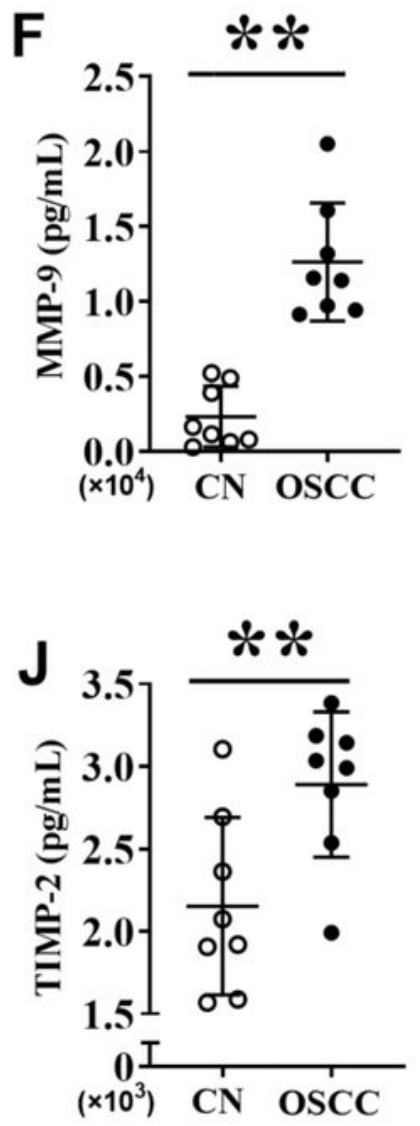

Figure 6 
(A) Representative protein array images showing the expression pattern of MMPs in the saliva of healthy controls and OCSS patients. Quantitative analysis of (B) MMP-1, (C) MMP-2, (D) MMP-3, (E) MMP-8, (F) MMP-9, (G) MMP-10, (H) MMP-13, (I) TIMP-1, and (J) TIMP-2 expression from protein array images. Data are presented as mean $\pm S D, n=8$. Significant differences compared with the healthy control group, ${ }^{*} p<0.05$ and ${ }^{* *} p<0.01$.



\section{Figure 7}

Correlation analysis of ANGs and MMPs expressions in HNSCC tumor tissues with cancer cell functional states. EMT: endothelial mesenchymal transition. 

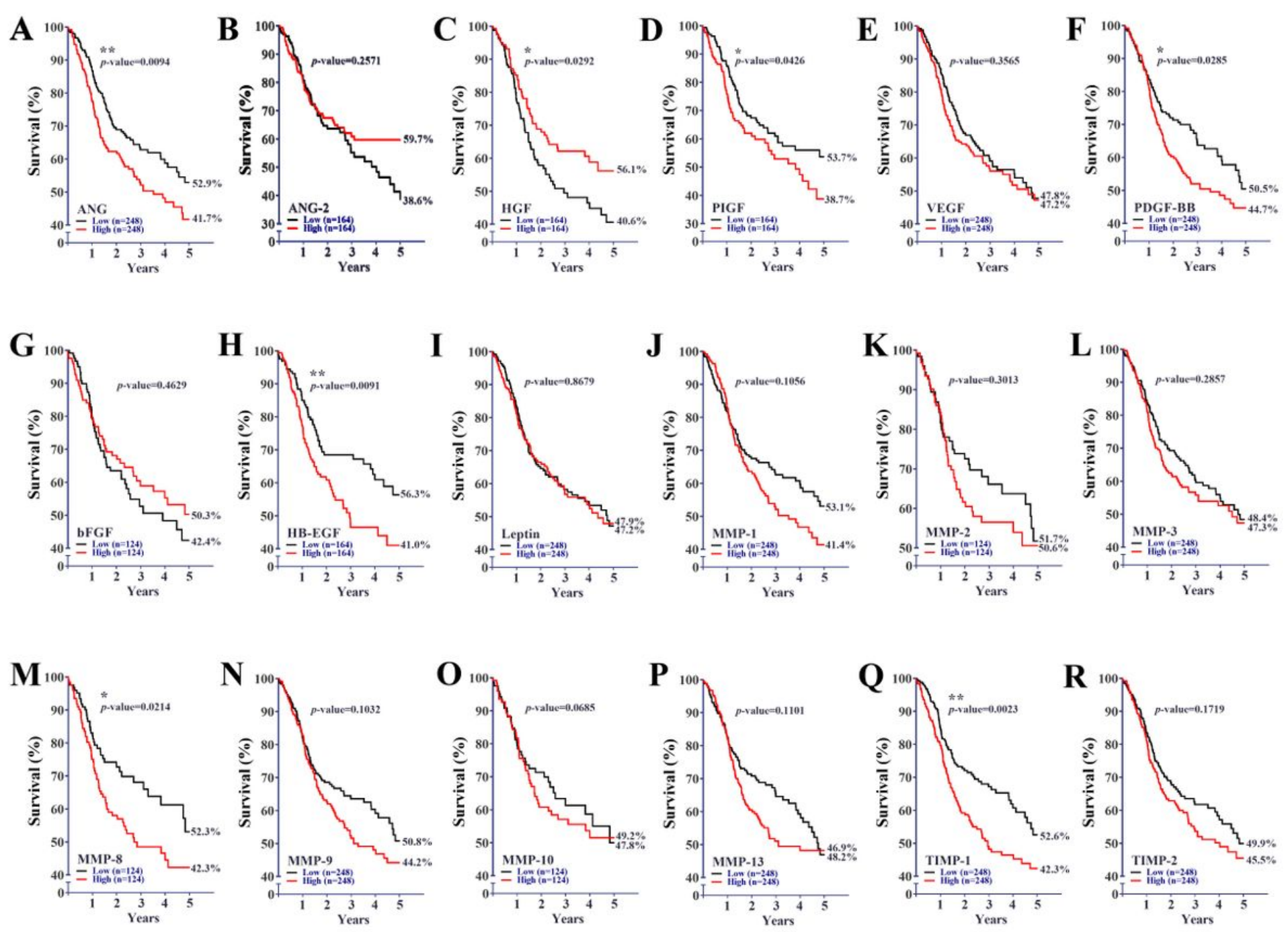

Figure 8

Correlation of (A) ANG, (B) ANG-2, (C) HGF, (D) PIGF, (E) VEGF, (F) PDGF-BB, (G) bFGF, (H) HB-EGF, (I) Leptin, (J) MMP-1, (K) MMP-2, (L) MMP-3, (M) MMP-8, (N) MMP-9, (O) MMP-10, (P) MMP-13, (Q) TIMP-1, or (R) TIMP-2 expression pattern with HNSC patient survival rate and duration (248 OSCC patients). ${ }^{*} p<0.05$ and ${ }^{* *} p<0.01$. 


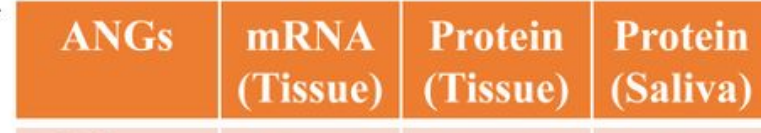

\begin{tabular}{|c|c|c|c|}
\hline ANG & $* * \uparrow$ & $* \uparrow$ & ns \\
\hline ANG-2 & $* * \uparrow$ & $* * \uparrow$ & ns \\
\hline HGF & $* \uparrow$ & $* * \uparrow$ & $* * \uparrow$ \\
\hline PLGF & $* * \uparrow$ & $* \uparrow$ & $* * \uparrow$ \\
\hline VEGF & $* * \uparrow$ & $* \uparrow$ & $* \uparrow$ \\
\hline PDGF-BB & $* \quad \uparrow$ & ns & $* \quad \uparrow$ \\
\hline bFGF & $* * \downarrow$ & ns & ns \\
\hline HB-EGF & $* * \uparrow$ & ns & ns \\
\hline Leptin & ns & ns & ns \\
\hline
\end{tabular}

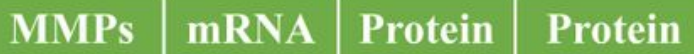 (Tissue)}

\begin{tabular}{|c|c|c|c|c|}
\hline ММР-1 & $*$ & $\uparrow$ & $*$ & $* *$ \\
\hline MMP-2 & $* *$ & $\uparrow$ & $* *$ & ns \\
\hline ММР-3 & $* *$ & $\uparrow$ & $*$ & $* * \uparrow$ \\
\hline MМР-8 & $* *$ & $\uparrow$ & $* *$ & $* * \uparrow$ \\
\hline ММР-9 & $* *$ & $\uparrow$ & $* *$ & $* * \uparrow$ \\
\hline ММР-10 & $*$ & $\uparrow$ & $*$ & $*$ \\
\hline ММР-13 & $* *$ & $\uparrow$ & $*$ & $*$ \\
\hline TIMP-1 & $* *$ & $\uparrow$ & $* *$ & ns \\
\hline TIMP-2 & $* *$ & $\uparrow$ & $* *$ & $* * 1$ \\
\hline
\end{tabular}



Figure 9

(A) ANGs and MMPs in the OSCC may serve as non-invasive diagnostic markers and therapeutic targets.

(B) The expression of ANGs in tissues and saliva. (C) The expression of MMPs in tissues and saliva. ${ }^{*} \mathrm{p}<0.05$ and ${ }^{* *} \mathrm{p}<0.01$. 
This is a list of supplementary files associated with this preprint. Click to download.

- TableS1.docx

- Tables2.docx 